\title{
HH 114 MMS: a new chemically active outflow ${ }^{\star}$
}

\author{
M. Tafalla ${ }^{1}$ and A. Hacar ${ }^{1,2}$ \\ ${ }^{1}$ Observatorio Astronómico Nacional (IGN), Alfonso XII 3, 28014 Madrid, Spain \\ e-mail: m.tafalla@oan.es \\ 2 Institute for Astrophysics, University of Vienna, Türkenschanzstrasse 17, 1180 Vienna, Austria \\ e-mail: alvaro.hacar@univie.ac.at
}

Received 15 February 2013 / Accepted 12 March 2013

\section{ABSTRACT}

\begin{abstract}
Context. A small group of bipolar protostellar outflows display strong emission from shock-tracer molecules such as $\mathrm{SiO}$ and $\mathrm{CH}_{3} \mathrm{OH}$, and are generally referred to as "chemically active". The best-studied outflow from this group is the one in L 1157.

Aims. We study the molecular emission from the bipolar outflow powered by the very young stellar object HH 114 MMS and compare its chemical composition with that of the L 1157 outflow.

Methods. We have used the IRAM 30 m radio telescope to observe a number of transitions from $\mathrm{CO}, \mathrm{SiO}, \mathrm{CH}_{3} \mathrm{OH}, \mathrm{SO}, \mathrm{CS}, \mathrm{HCN}$, and $\mathrm{HCO}^{+}$toward the $\mathrm{HH} 114$ MMS outflow. The observations consist of maps and a two-position molecular survey.

Results. The HH 114 MMS outflow presents strong emission from a number of shock-tracer molecules that dominate the appearance of the maps around the central source. The abundance of these molecules is comparable to the abundance in L 1157. Conclusions. The outflow from HH 114 MMS is a spectacular new case of a chemically active outflow.
\end{abstract}

Key words. stars: formation - ISM: abundances - ISM: jets and outflows - ISM: individual objects: HH 114 MMS - ISM: molecules radio lines: ISM

\section{Introduction}

Bipolar outflows from young stellar objects (YSOs) are routinely identified by their mechanical impact on the surrounding cloud, which produces characteristic lobes of blue- and redshifted material. A small group of outflows also show evidence of chemical impact in the form of enhanced abundance of shocksensitive species such as $\mathrm{SiO}$ and $\mathrm{CH}_{3} \mathrm{OH}$. These outflows are often referred to as "chemically active," and their study offers a unique opportunity to investigate both ambient gas acceleration and the complex reactions of shock chemistry (Tafalla \& Bachiller 2011).

Here we report the serendipitous identification of a chemically active outflow driven by HH 114 MMS. HH 114 MMS is located in the L 1589 cloud, at an estimated distance of $450 \mathrm{pc}$ (Mathieu 2008). It was discovered at mm-wavelengths by Chini et al. (1997), and was later mapped at cm wavelengths by Rodríguez \& Reipurth (1996). Both HH 114 MMS and the neighboring source IRAS $05155+0707$ lie near the center of the 2.6-pc long chain of Herbig-Haro (HH) objects HH 114/HH 115 (Reipurth et al. 1997), although the relation between the two YSOs and the $\mathrm{HH}$ chain remains unclear. Each YSO drives a separate $\mathrm{CO}$ outflow, and there is no evidence for any interaction between the two. As shown by Lee et al. (2002), the blue lobe of the HH 114 MMS outflow approximately coincides with HH 114D, but the large-scale orientation of the HH 114/HH 115 system differs significantly from the direction of the two CO outflows. While it is possible that IRAS $05155+0707$ drives the large-scale $\mathrm{HH}$ chain (if its initially $\mathrm{E}-\mathrm{W}$ direction changes to

^ Based on observations carried out with the IRAM 30 m Telescope. IRAM is supported by INSU/CNRS (France), MPG (Germany) and IGN (Spain).
SE-NW), it is unlikely that the HH 114 MMS outflow plays any role in the large-scale HH 114/HH 115 system, since that would require a change of outflow direction close to 90 degrees.

HH 114 MMS is a highly embedded object. Chini et al. (1997) suggested it is a class 0 object (André et al. 1993), and determined an upper limit to its luminosity of about $25 L_{\odot}$. Further evidence for its extreme youth comes from the high collimation of its molecular outflow shown by the interferometric maps of Arce \& Sargent (2006).

\section{Observations}

The HH 114 MMS bipolar outflow was observed in the $\mathrm{CO}(2-1)$ line with the IRAM 30 m telescope during December 2008 using the dual-polarization $3 \times 3$ HERA array operating in wobblerswitching mode with a throw of $240^{\prime \prime}$ and a frequency of $0.5 \mathrm{~Hz}$. The signal from HERA was sent to the VESPA autocorrelator, which was configured to produce spectra with a resolution of $1.25 \mathrm{MHz}\left(1.6 \mathrm{~km} \mathrm{~s}^{-1}\right)$ over a $280 \mathrm{MHz}\left(365 \mathrm{~km} \mathrm{~s}^{-1}\right)$ passband. These spectra were later processed with the CLASS software ${ }^{1}$ and converted to the main beam brightness temperature scale.

Additional IRAM 30 m observations of the HH 114 MMS outflow were made in February 2011. An initial survey for selected molecular lines in the 1-3 mm wavelength bands was carried out toward several positions. In view of the bright lines of shock-tracer species, additional positions were observed to map the full extent of the emission. These shock-tracer observations were complemented with limited maps of the quiescentgas tracers $\mathrm{N}_{2} \mathrm{H}^{+}(1-0)$ and $\mathrm{C}^{18} \mathrm{O}(2-1)$. All observations were made with the dual-band, dual-polarization, single-pixel EMIR

1 http://www.iram.fr/IRAMFR/GILDAS 
A\&A 552, L9 (2013)
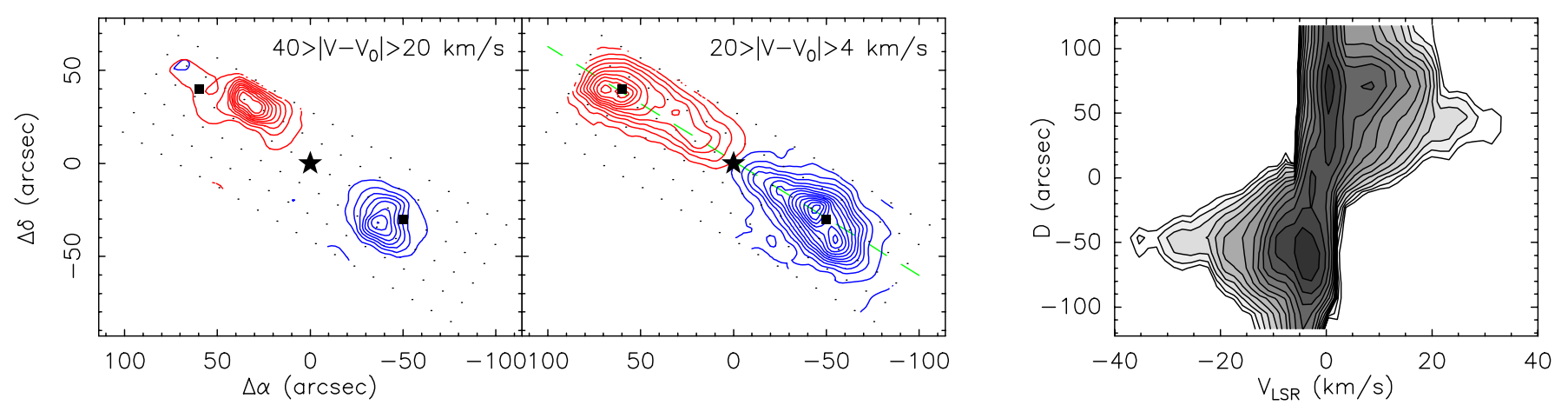

Fig. 1. Structure of the HH 114 MMS outflow from $\mathrm{CO}(2-1)$ data. Left: maps of intensity integrated in velocity ranges symmetrically separated from the ambient cloud velocity $\left(V_{0}=-0.5 \mathrm{~km} \mathrm{~s}^{-1}\right.$ from $\mathrm{N}_{2} \mathrm{H}^{+}$data). First contour and contour interval are $1 \mathrm{~K} \mathrm{~km} \mathrm{~s}$ in the high-velocity map and $5 \mathrm{~K} \mathrm{~km} \mathrm{~s}^{-1}$ in the low-velocity map. The star symbol at the offset origin represents the position of HH $114 \mathrm{MMS}\left(\alpha(\mathrm{J} 2000)=05^{\mathrm{h}} 18^{\mathrm{m}} 15^{\mathrm{s}} .2\right.$, $\left.\delta(\mathrm{J} 2000)=+7^{\circ} 12^{\prime} 2^{\prime \prime}\right)$, the two squares are the positions where the line survey was carried out, and the dots are observed positions. The dashed green line is the direction of the position-velocity diagram shown in the next panel. Right: position-velocity diagram along the outflow axis. To emphasize the low-level outflow emission, the contours are spaced by factors of square root of 2 starting at $0.1 \mathrm{~K}$.

receiver. For the simultaneous $\mathrm{N}_{2} \mathrm{H}^{+}$and $\mathrm{C}^{18} \mathrm{O}$ observations, we used frequency-switching mode to ensure that the spectra were free from ambient-cloud contamination. For the rest of the observations, we used the same wobbler-switching mode used in the $\mathrm{CO}(2-1)$ observations. As spectrometers, we used the FTS and VESPA instruments, which, depending on the line, provided velocity resolutions between 0.06 and $0.7 \mathrm{~km} \mathrm{~s}^{-1}$. The resulting spectra were processed with CLASS as done with the $\mathrm{CO}(2-1)$ spectra.

\section{Physical properties of the HH 114 MMS outflow}

Figure 1 summarizes the main features of the $\mathrm{CO}(2-1)$ data. The two maps in the left and middle panels show that the $\mathrm{CO}$ emission has a markedly bipolar distribution with respect to HH 114 MMS, with the redshifted emission toward the northeast and the blueshifted emission toward the southwest. This distribution agrees with that found by Arce \& Sargent (2006) from interferometric observations of the central arcminute around the YSO, and with the distribution of blue $\mathrm{CO}$ emission mapped by Lee et al. (2002), also with an interferometer (and restricted to the southern blue lobe). Our more extended $\mathrm{CO}(2-1)$ maps show that the HH 114 MMS outflow has a high degree of collimation at all velocities, and that its emission extends for at least $200^{\prime \prime}$ $(0.4 \mathrm{pc}$ for the assumed distance of $450 \mathrm{pc}$ ).

To illustrate the velocity field of the gas, we present a position-velocity diagram along the outflow axis in the rightmost panel of Fig. 1. This diagram shows that both outflow lobes follow an approximate linear increase of the terminal velocity with distance from the central source. The "Hubble-law" velocity pattern, however, is only approximate, since the fastest velocities are not reached at the end of the lobes but at an intermediate distance. This can also be seen in the velocity maps of Fig. 1, which show that the gas faster than $20 \mathrm{~km} \mathrm{~s}^{-1}$ forms two bright spots about $50^{\prime \prime}$ from the YSO. The origin of this velocity pattern is unclear, and the 1-beam sampling of our data is too coarse to allow further investigation. The blue-red symmetry of the pattern, however, suggests that it is intrinsic to the outflow, and not the result of an interaction with the ambient cloud. While unusual, the pattern seems not unique to HH 114 MMS. Similar cases, together with their difficult interpretation in terms of existing outflow models, have been discussed by Chernin \& Masson (1995).

We complete our analysis estimating the energetics of the moving gas traced by $\mathrm{CO}(2-1)$. We follow standard practice and do not correct for the uncertain inclination angle of the outflow. The lack of overlap between the blue and red lobes, together with the lack of color mixing inside each lobe, make HH 114 MMS a Case 2 outflow in the classification of Cabrit \& Bertout (1986), suggesting an inclination angle between $15^{\circ}$ and $75^{\circ}$ (we estimate an outflow opening angle $\theta_{\max }=15^{\circ}$ from the CO maps). As a result, the inclination correction to the velocity lies between $4 \%$ and a factor of 4 , and this makes our energetics estimate a strict lower limit. To avoid contamination from ambientcloud material, we also limit our analysis to emission faster than $3 \mathrm{~km} \mathrm{~s}^{-1}$. We also assume that the $\mathrm{CO}(2-1)$ emission is optically thin and in local thermodynamic equilibrium (LTE), with an excitation temperature of $25 \mathrm{~K}$ (from the $\mathrm{CO}(3-2) / \mathrm{CO}(2-1)$ ratio, Sect. 5), and use a CO abundance of $8.5 \times 10^{-5}$ (Frerking et al. 1982).

With these assumptions, we estimate a total outflow mass of $0.15 M_{\odot}$, a momentum of $1.3 M_{\odot} \mathrm{km} \mathrm{s}^{-1}$, and a kinetic energy of $3.5 \times 10^{44} \mathrm{erg}$. These values are between four times less than (mass and momentum) and equal to (energy) those estimated for L 1157 by Bachiller et al. (2001), who do not correct for inclination in this also Case 2 outflow.

\section{Maps of dense-gas tracers}

While the CO maps of the HH 114 MMS outflow are not qualitatively different from those of other young outflows (e.g., Lee et al. 2002; Arce \& Sargent 2006), the maps of the high-density tracers are truly remarkable. Figure 2 shows that the emission of dense gas tracers such as $\mathrm{HCN}, \mathrm{CS}, \mathrm{SO}, \mathrm{CH}_{3} \mathrm{OH}$, or $\mathrm{SiO}$ is not concentrated toward the central source, but presents two bright peaks toward the outflow lobes. $\mathrm{HCO}^{+}$, while centrally concentrated, also shows an elongation in the direction of the outflow, and only $\mathrm{N}_{2} \mathrm{H}^{+}$and $\mathrm{C}^{18} \mathrm{O}$ (the last one not strictly a high-density tracer) present compact emission peaks towards HH 114 MMS. (The southern $\mathrm{N}_{2} \mathrm{H}^{+}$and $\mathrm{C}^{18} \mathrm{O}$ peak coincides with the vicinity of IRAS 05155+0707.)

The similarity between the emission of the dense-gas tracers and the $\mathrm{CO}$ outflow is not limited to the integrated maps of Fig. 2. Velocity maps using the intervals of Fig. 1 show the same blue-red bipolar distribution and the same offset between highand low-velocity peaks. Individual spectra, like those in Fig. 3, show prominent wings of clear outflow origin.

Bipolar outflows are known to sometimes enhance the emission of certain molecular species, especially $\mathrm{SiO}$ and $\mathrm{CH}_{3} \mathrm{OH}$, 
M. Tafalla and A. Hacar: HH 114 MMS: a new chemically active outflow

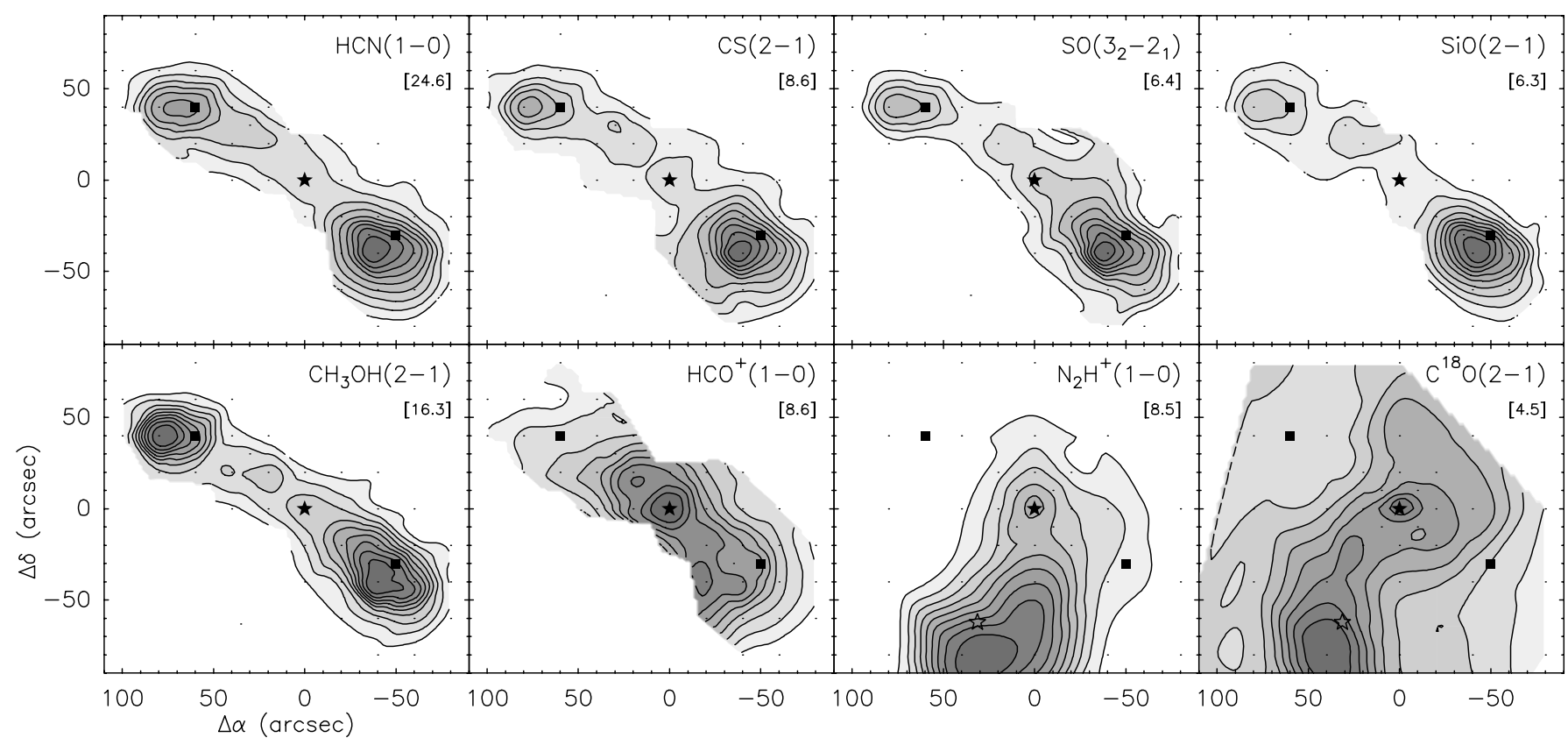

Fig. 2. Emission from dense-gas tracers in the vicinity of HH 114 MMS. The maps represent emission integrated over the complete velocity range of detection. Contours are at $10,20, \ldots 90 \%$ of the map maximum, whose value in $\mathrm{K} \mathrm{km} \mathrm{s}^{-1}$ is given within brackets under the name of the transition. Central positions, dots, and solid star as in Fig. 1. The open star in the last two panels represents IRAS $05155+0707$.

due to the disruption of dust grains and the ices that coat them (van Dishoeck \& Blake 1998). Few outflows, however, display the dramatic impact seen in the emission of other tracers associated with dense core gas, such as CS and HCN. These species are routinely used to trace the innermost environment around YSOs (e.g., Afonso et al. 1998; Launhardt et al. 1998), and are not expected to represent outflow gas. As Fig. 2 shows, this is not the case in HH 114 MMS, where the core emission is dwarfed by the outflow contribution.

As mentioned before, only a small group of outflows present the prominent emission from dense-gas tracers found towards HH 114 MMS. The best-known member of this group is the L 1157 outflow, which has long been recognized for presenting unusually strong emission from shock-tracer molecules, such as $\mathrm{SiO}$ (Mikami et al. 1992). The molecular maps of Bachiller et al. (2001) (their Fig. 6) show that most dense-gas tracers in $\mathrm{L} 1157$ (including $\mathrm{HCN}, \mathrm{CS}, \mathrm{SO}, \mathrm{CH}_{3} \mathrm{OH}$, and $\mathrm{SiO}$ ) present a remarkable pattern of outflow-dominated emission, similar to that shown in Fig. 2 for HH 114 MMS.

The dramatic effect of the L 1157 outflow in the chemistry of its gas led Bachiller et al. (2001) to coin the term "chemically active" to refer to this outflow and others with similar properties, such as BHR 71 (Bourke et al. 1997; Garay et al. 1998). The maps of Fig. 2 show now that the term can also be applied to the HH 114 MMS outflow, which appears as one of the finest examples of this class, thanks to its clean pattern of a single bright emission peak near the end of two lobes of similar size.

\section{Column density and abundance estimates}

To further characterize the chemistry of the HH 114 MMS outflow, we convert the observed intensities into molecular column densities. For this, we use the results of a small survey of transitions at $1-3 \mathrm{~mm}$ wavelength toward $\left(60^{\prime \prime}, 40^{\prime \prime}\right)$ and $\left(-50^{\prime \prime}\right.$, $\left.-30^{\prime \prime}\right)$. Representative spectra from this survey are shown in Fig. 3. The full list of detections include $\mathrm{CO}(2-1), \mathrm{CO}(3-2)$, $\mathrm{CS}(2-1), \mathrm{CS}(3-2), \mathrm{SiO}(2-1), \mathrm{SiO}(3-2), \mathrm{SO}\left(3_{2}-21\right), \mathrm{SO}\left(4_{3}-3_{2}\right)$,
$\mathrm{CH}_{3} \mathrm{OH}\left(2_{k}-1_{k}\right), \mathrm{CH}_{3} \mathrm{OH}\left(3_{k}-2_{k}\right), \mathrm{HCN}(1-0), \mathrm{HCO}^{+}(1-0)$, and $\mathrm{H}_{2} \mathrm{CO}\left(2_{02}-1_{01}\right)$.

At the time of the observations, and based on our CO maps, the two survey positions were thought to represent the brightest peaks of each outflow lobe. Later mapping in multiple molecules, however, showed that these positions were offset from the true emission peaks by $\sim 15^{\prime \prime}$. While these small offsets are not critical for the abundance results, which seem almost position-independent, they may affect the comparison of the HH 114 MMS outflow intensities with those of other objects.

As a first step in our column density estimate we chose for each survey position a $20 \mathrm{~km} \mathrm{~s}^{-1}$-wide interval of outflow velocities (see values in Table 1). The molecular emission in this interval was analyzed with an LTE method similar to that presented in Tafalla et al. (2010), although no dilution factors were applied to the data because the observations were not centered on the emission peak. For the $\mathrm{CO}$ emission, we used data from 5-point crosses with a $5^{\prime \prime}$ sampling to convolve the $\mathrm{CO}(3-2)$ emission to the $12^{\prime \prime}$ resolution of the $\mathrm{CO}(2-1)$ data. These two intensities were used to derive $\mathrm{CO}$ excitation temperatures between 25 and $30 \mathrm{~K}$ and the column density values reported in Table 1 . For the dense-gas tracers with two observed transitions (CS, SiO, SO, and $\mathrm{CH}_{3} \mathrm{OH}$ ), we used population diagrams to determine simultaneously column densities and excitation temperatures, which range from about 4 to $8 \mathrm{~K}$ and indicate that the molecules are subthermally excited. Finally, for the three species with only one observed transition $\left(\mathrm{HCN}, \mathrm{HCO}^{+}\right.$, and para- $\mathrm{H}_{2} \mathrm{CO}$ ), we assumed an excitation temperature of $6 \mathrm{~K}$ (mean value of $\mathrm{CS}, \mathrm{SiO}$, $\mathrm{SO}$, and $\mathrm{CH}_{3} \mathrm{OH}$ ), and used the integrated intensities to estimate molecular column densities.

Using the previous column densities, we determined for each species an abundance with respect to $\mathrm{CO}$, and compared this value with the typical undepleted value in a dense core as discussed in Tafalla et al. (2010). The results of this estimate are presented in Table 1 in terms of the enhancement factor $f_{\text {enh }}$, which is the ratio between the outflow and the core CO-normalized abundances. As can be seen, the $f_{\text {enh }}$ factors in 


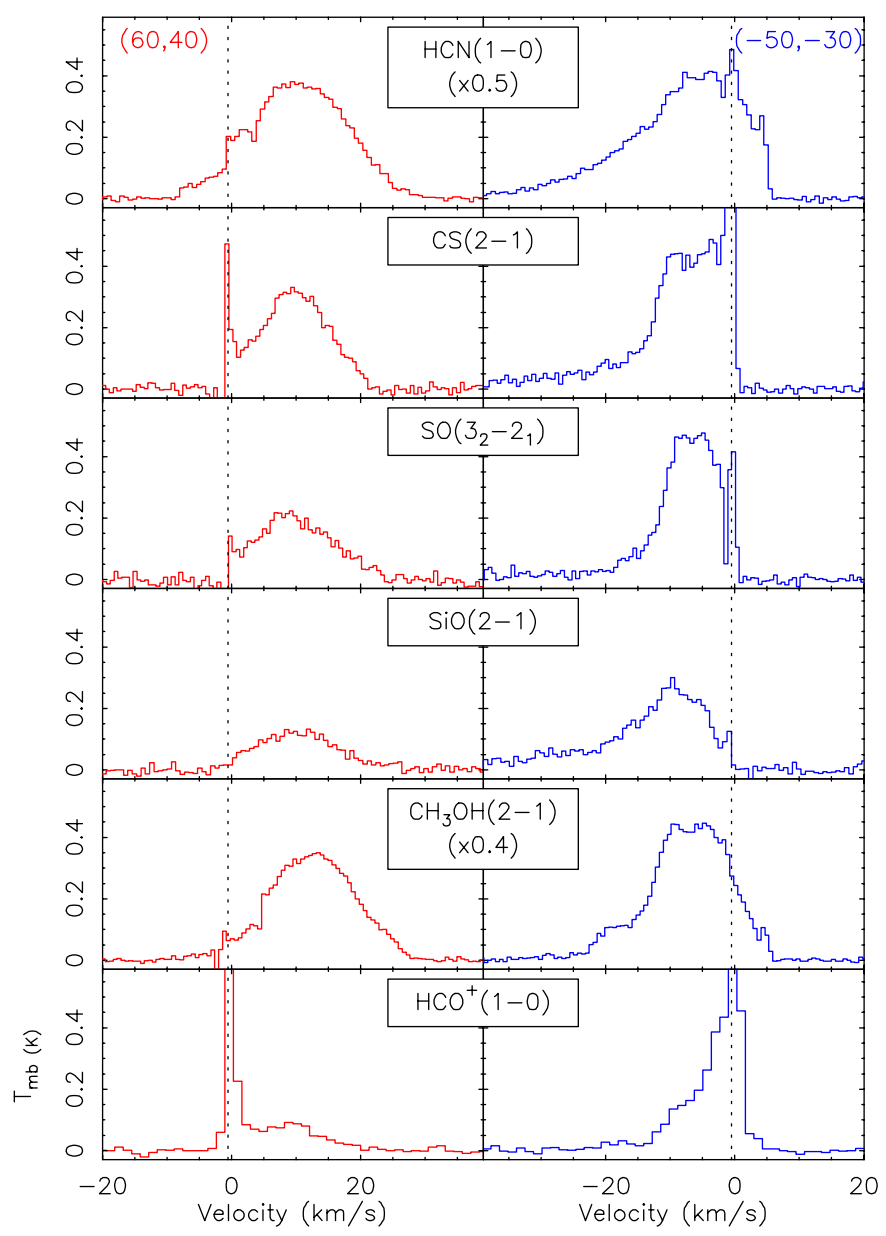

Fig. 3. Sample spectra from the line survey carried out toward two outflow positions. The offset of each position is indicated in the top panel (in arcseconds and referred to the map center of Fig. 1). The vertical dashed line indicates the velocity of the ambient cloud.

the two outflow positions agree with each other within a factor of 2 for all observed species. This agreement, which is probably at the level of our measurement uncertainty, suggests that the two outflow lobes are chemically very similar.

The results in Table 1 also show that the enhancement factors of the different species span a wide range of values. The smallest factor is that of $\mathrm{HCO}^{+}$, and is consistent with this molecule having little or no enhancement in the outflow gas. This result is consistent with the map in Fig. 2, which shows no emission peak for this molecule toward neither of the outflow lobes (although the emission is elongated in the outflow direction). All other species in Table 1 present significant abundance enhancements, and range from about one order of magnitude in the case of $\mathrm{HCN}$ and CS to more than three orders of magnitude in the case of $\mathrm{CH}_{3} \mathrm{OH}$ and $\mathrm{SiO}$.

While large, the enhancement factors in Table 1 are comparable to those in other young outflows. This can be seen by comparing the values in the table with those presented by Tafalla et al. (2010), who measured enhancement factors in the L 1448 and IRAS 04166 outflows and re-evaluated the L 1157-B1 estimates previously made by Bachiller \& Pérez Gutiérrez (1997). The values for these three outflows are in good agreement with our HH 114 MMS estimates, and for example, the geometrical mean of the $f_{\text {enh }}$ estimates in the two HH 114 MMS survey positions agrees with those in L 1157-B1 better than a factor of 3 for all molecular species. Such an agreement in enhancement
Table 1. Survey column densities and abundance enhancements.

\begin{tabular}{|c|c|c|c|c|}
\hline \multirow[b]{2}{*}{ MOL } & \multicolumn{2}{|c|}{$\left(60^{\prime \prime}, 40^{\prime \prime}\right)^{a}$} & \multicolumn{2}{|c|}{$\left(-50^{\prime \prime},-30^{\prime \prime}\right)^{b}$} \\
\hline & $N\left(\mathrm{~cm}^{-2}\right)$ & $f_{\mathrm{enh}}^{c}$ & $N\left(\mathrm{~cm}^{-2}\right)$ & $f_{\mathrm{enh}}^{c}$ \\
\hline $\mathrm{CO}$ & $1.8 \times 10^{16}$ & - & $2.1 \times 10^{16}$ & - \\
\hline $\mathrm{HCO}^{+}$ & $1.0 \times 10^{12}$ & 1.1 & $1.1 \times 10^{12}$ & 1.1 \\
\hline $\mathrm{HCN}$ & $2.1 \times 10^{13}$ & 11 & $1.6 \times 10^{13}$ & 7.2 \\
\hline $\mathrm{CS}$ & $1.8 \times 10^{13}$ & 14 & $2.8 \times 10^{13}$ & 19 \\
\hline $\mathrm{p}-\mathrm{H}_{2} \mathrm{CO}$ & $8.2 \times 10^{12}$ & 65 & $8.9 \times 10^{12}$ & 61 \\
\hline SO & $3.4 \times 10^{13}$ & 290 & $4.4 \times 10^{13}$ & 320 \\
\hline $\mathrm{CH}_{3} \mathrm{OH}$ & $4.6 \times 10^{14}$ & $1.8 \times 10^{3}$ & $3.2 \times 10^{14}$ & $1.1 \times 10^{3}$ \\
\hline $\mathrm{SiO}$ & $5.5 \times 10^{12}$ & $5.1 \times 10^{3}$ & $1.4 \times 10^{13}$ & $1.1 \times 10^{4}$ \\
\hline
\end{tabular}

Notes. ${ }^{(a)}$ Quantities measured in the $V_{\mathrm{LSR}}$ range 4 to $24 \mathrm{~km} \mathrm{~s}^{-1}$. (b) Quantities measured in the $V_{\mathrm{LSR}}$ range -26 to $-6 \mathrm{~km} \mathrm{~s}^{-1}$. (c) Abundance enhancement factor over dense core value as in Tafalla et al. (2010).

factors confirms the idea that HH 114 MMS must be a bona fide chemically active outflow.

Although the abundances with respect to $\mathrm{CO}$ in the HH 114 MMS outflow are comparable to those in L 1157, the integrated intensities towards its brightest molecular peak are lower than those of L 1157-B1 by an average factor of 2.5 . This suggests that the HH 114 MMS outflow may not have accumulated as much enriched material as L 1157, although beam dilution may still affect the HH 114 MMS intensities. The more southern location of HH 114 MMS, on the other hand, makes this outflow a good candidate for ALMA observations, something impossible with L 1157 due to its high declination. The newly-identified chemically active HH 114 MMS outflow therefore has significant potential to shed new light on the stillmysterious nature of this outflow class.

Acknowledgements. We thank the observers of the HERA pool for obtaining the $\mathrm{CO}(2-1)$ data, and the IRAM staff for help during the EMIR observations. The anonymous referee and Malcolm Walmsley provided a number of useful comments. M.T. acknowledges support from the MICINN program CONSOLIDER INGENIO 2010, grant "Molecular Astrophysics: The Herschel and ALMA era ASTROMOL" (ref.: CSD2009-00038). This publication is also supported by the Austrian Science Fund (FWF). This research has made use of NASA's Astrophysics Data System Bibliographic Services together with the SIMBAD database and the VizieR catalogue access tool operated at CDS, Strasbourg, France.

\section{References}

Afonso, J. M., Yun, J. L., \& Clemens, D. P. 1998, AJ, 115, 1111 André, P., Ward-Thompson, D., \& Barsony, M. 1993, ApJ, 406, 122

Arce, H. G., \& Sargent, A. I. 2006, ApJ, 646, 1070

Bachiller, R., \& Pérez Gutiérrez, M. 1997, ApJ, 487, L93

Bachiller, R., Pérez Gutiérrez, M., Kumar, M. S. N., \& Tafalla, M. 2001, A\&A, 372,899

Bourke, T. L., Garay, G., Lehtinen, K. K., et al. 1997, ApJ, 476, 781

Cabrit, S., \& Bertout, C. 1986, ApJ, 307, 313

Chernin, L. M., \& Masson, C. R. 1995, ApJ, 455, 182

Chini, R., Reipurth, B., Sievers, A., et al. 1997, A\&A, 325, 542

Frerking, M. A., Langer, W. D., \& Wilson, R. W. 1982, ApJ, 262, 590

Garay, G., Köhnenkamp, I., Bourke, T. L., Rodríguez, L. F., \& Lehtinen, K. K. 1998, ApJ, 509, 768

Launhardt, R., Evans, N. J., II, Wang, Y., et al. 1998, ApJS, 119, 59

Lee, C.-F., Mundy, L. G., Stone, J. M., \& Ostriker, E. C. 2002, ApJ, 576, 294

Mathieu, R. D. 2008, Handbook of Star Forming Regions, 1, 757

Mikami, H., Umemoto, T., Yamamoto, S., \& Saito, S. 1992, ApJ, 392, L87

Reipurth, B., Bally, J., \& Devine, D. 1997, AJ, 114, 2708

Rodríguez, L. F., \& Reipurth, B. 1996, Rev. Mex. Astron. Astrofis., 32, 27

Tafalla, M., \& Bachiller, R. 2011, IAU Symp., 280, 88

Tafalla, M., Santiago-García, J., Hacar, A., \& Bachiller, R. 2010, A\&A, 522, A91

van Dishoeck, E. F., \& Blake, G. A. 1998, ARA\&A, 36, 317 